\title{
Cecal diverticulitis mimicking as appendicitis
}

\section{KEYWORDS: computed tomography}

Diverticulum of the cecum is relatively rare and generally is benign and asymptomatic. However, inflammation of a diverticulum may present with symptoms related to appendicitis. 72 y old male presented to the ED with abdominal pain and nausea. Physical examination revealed tenderness in the right lower quadrant. Subsequent CT scan revealed inflammatory stranding adjacent to the cecum centered around the diverticula. Several foci of air along the adjacent mesentery (FIGURE 1). The appendix is normal in caliber. Final diagnoses of cecal diverticulitis with macroperforation was made. Patient was treated with IV ciprofloxacin and flagyl. His symptoms gradually improved.

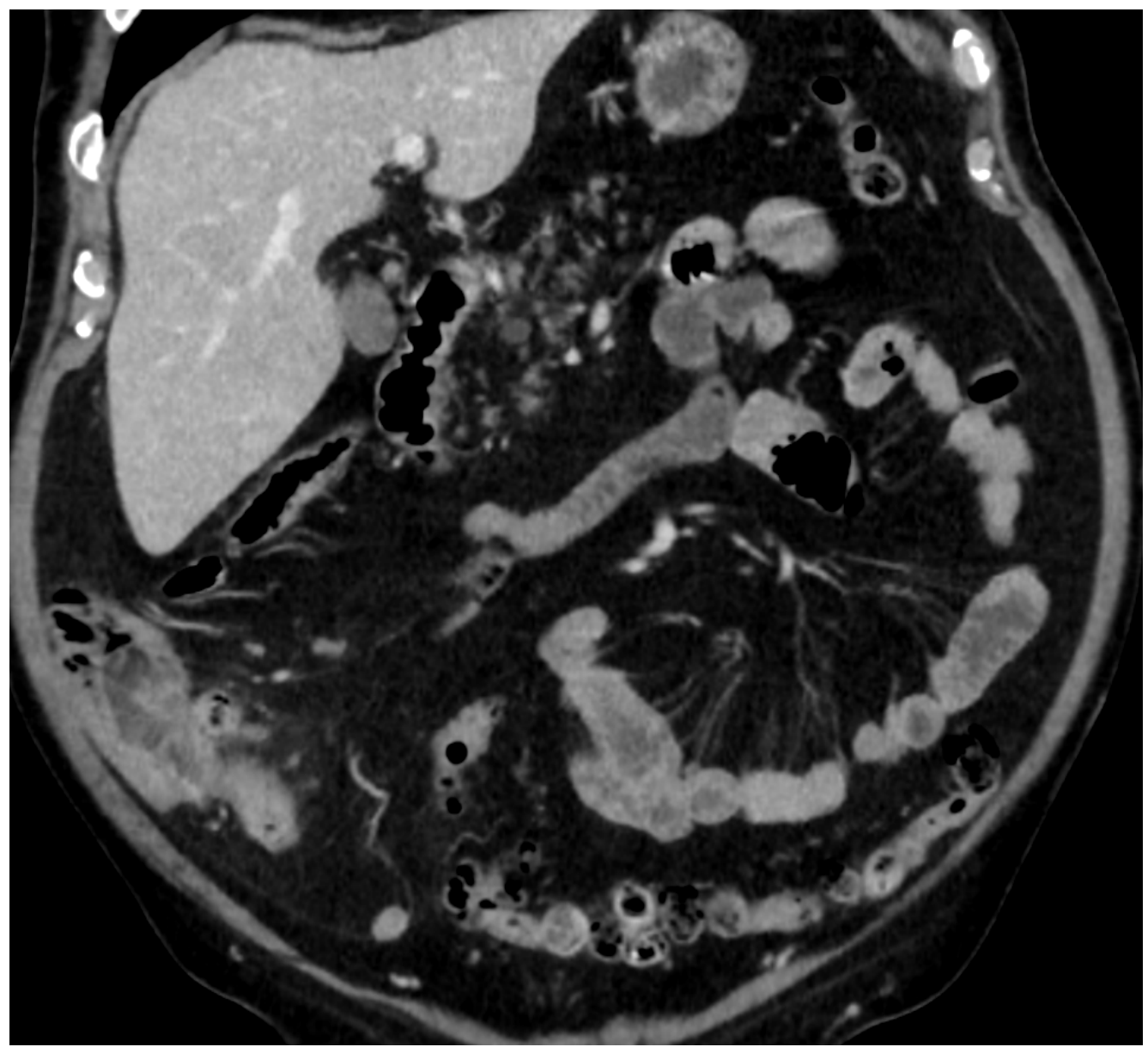

Figure 1. CT scan of abdomen showing inflammatory stranding in the right lower quadrant adjacent to the medial border of cecum, centered around a diverticulum suggestive of cecal diverticulitis. 